\title{
Increasing paintball related eye trauma reported to a state eye injury registry
}

\author{
John W Kitchens, Ronald P Danis
}

\begin{abstract}
Objectives-To evaluate an apparent increase in documented trauma from paintball related eye injuries reported to the Eye Injury Registry of Indiana.

Methods-A retrospective review of cases reported to the database is reported, with representative case histories.

Results-No injuries from paintball were reported during the period June 1992 to June 1996. Over the next two years 11 injuries were reported, representing $4 \%$ of all ocular trauma reports over this period. Visual outcome is poor in many of these eyes and more than one half present with posterior segment ocular injury.

Conclusions-Severe ocular trauma results from impacts from paintball pellets, and the occurrence of injuries appears to be increasing due to growth in popularity of this war game. Diligent use of eye protection by all participants is necessary to prevent a continuing rise in ocular trauma prevalence from this activity.

(Injury Prevention 1999;5:301-302)
\end{abstract}

Keywords: paintball; eye trauma; injury registry

Paintball is a rapidly growing sport introduced 18 years ago and currently played in over 40 countries by millions of participants. ${ }^{1}$ In it players try to eliminate opponents from the game by shooting them with paintballs from compressed air or gas rifles ${ }^{1}$ at a muzzle velocity of $80-130 \mathrm{~mm} / \mathrm{sec}^{2}$ Paintballs are $14 \mathrm{~mm}$ projectiles containing water, glycerin, polyethylene glycol, titanium oxide, and dyes within a gelatin or latex coat. ${ }^{2}$ Upon impact, the ball coat ruptures, releasing the colored liquid to mark the area of impact. Over a two year period, there were 11 reported cases of severe ocular injury to the Eye Injury Registry of Indiana (EIRIN), whereas none were reported before 1996.

Department of Ophthalmology, Indiana University School of Medicine J W Kitchens R P Danis

Correspondence to: Dr Ronald P Danis, Department of Ophthalmology, 702 Rotary Circle, Indianapolis, IN 46202, USA (e-mail: rdanis@iupui.edu)

\section{Methods}

EIRIN was established in June 1992, sponsored by the Indiana Academy of Ophthalmology, as an affiliate of the United States Eye Injury Registry. Forms are provided to ophthalmologists throughout Indiana. Data from the forms are entered on FileMaker Pro, which has a search engine for text words and diagnosis. Through August 1998, the registry received over 909 cases of severe eye injuries reported from ophthalmologists throughout the state (an average of 151 cases annually).
Results

There were 11 reported cases of eye injuries resulting from paintball between June 1996 and August 1998; this is over 4\% of the 264 cases of severe ocular injury reported to EIRIN over this period. Of these, all patients were male with a mean age of 22 (range 9-41) years. Only two were wearing eyewear, and none were wearing protective safety glasses or facemasks. Ten of the cases were described as accidental with one reported as an assault. The most common injuries included bleeding into the anterior chamber (hyphema; $n=7$ ), bleeding into the vitreous cavity (vitreous hemorrhage; $\mathrm{n}=6$ ), retinal hemorrhage, and retinal edema $(\mathrm{n}=3$ each). Visual acuity on initial examination was reported as worse than $4 / 200$ in seven of the cases with vision reduced to the level of detecting only hand motion in five. Four of the cases required urgent surgical intervention for management of these complications.

\section{Case reports}

CASE 1

A 17 year old male was unintentionally shot in his right eye while playing unsupervised in a backyard paintball game without protective eyewear. Initial examination revealed a visual acuity of $4 / 200$, a $30 \%$ hyphema, vitreous hemorrhage, choroidal rupture, and an afferent pupillary defect. On six month follow up, his best corrected visual acuity remained 4/200 with severe visual field loss.

\section{CASE 2}

A 26 year old male was struck in an unprotected right eye by a paintball pellet after he removed his protective facemask to clean it. Initial examination revealed reduced visual acuity to hand movements only, hyphema, vitreous hemorrhage, and choroidal rupture. One month after the injury he had surgery, a posterior vitrectomy, for the vitreous hemorrhage. Postoperative acuity improved to $20 / 200$ at six months with severe visual field loss.

\section{Discussion}

Ocular injuries observed from paintball are typical for severe blunt trauma. Seven out of 11 ocular paintball injuries in our series involved posterior segment trauma (injury to the retina, vitreous cavity and/or choroid). This is a similar proportion of injured eyes as in other published series. ${ }^{3-5}$ A comprehensive literature review revealed a large proportion of paintball related injuries involved retinal damage (36 of 76 ), vitreous hemorrhage (22 of 76), and choroidal rupture (five of 76 ). ${ }^{2}$ In this review, 


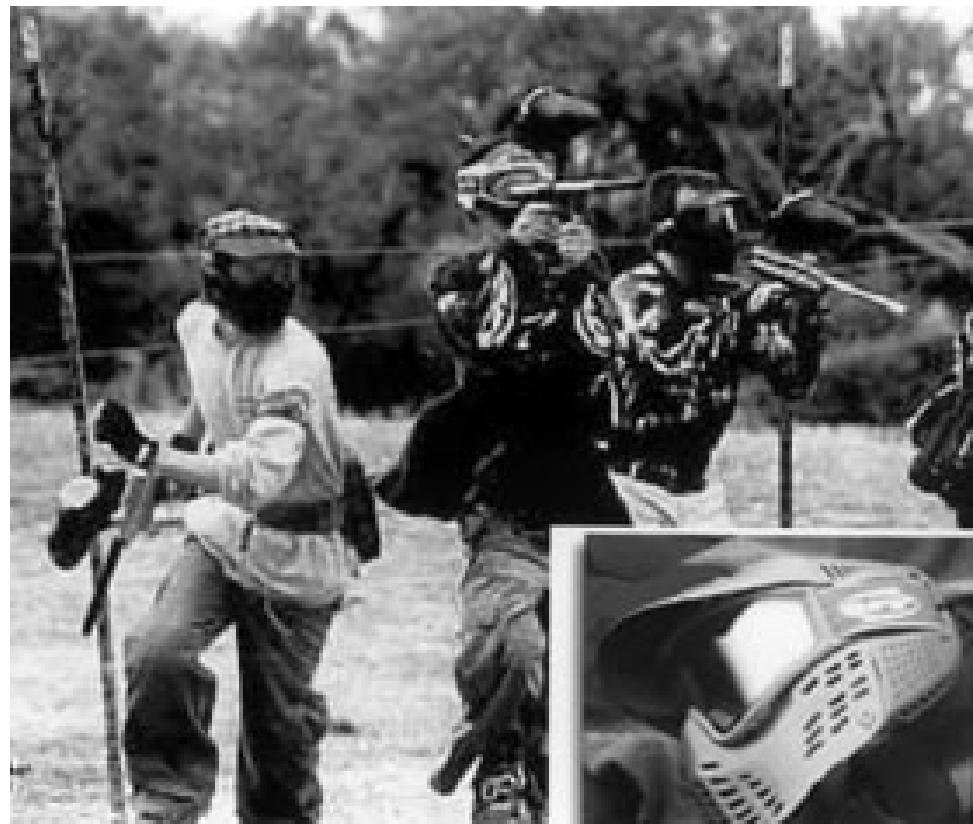

Figure 1 Paintball players employing appropriate facial protection. Inset: example of paintball facemask. The American Society of Testing and Materials has established manufacturer guidelines for $2.5 \mathrm{~mm}$ minimum thickness polycarbonate lenses to be used for paintball. $^{7}$

31 of 76 patients demonstrated a final visual acuity of $20 / 200$ or worse. Another recent report of injuries from paintball treated in tertiary referral centers reported that nine of 13 patients suffered posterior segment injury, and eight patients had final acuity of $20 / 200$ or worse. ${ }^{5}$ The major risk factors for poor vision outcome are posterior segment injury, and poor initial visual acuity. ${ }^{6}$

The significance of this report lies in that data were collected from a statewide registry that has had roughly equal numbers of injuries collected annually for six years of operation, yet paintball injuries only began to appear in the last two years of analysis. These injuries now constitute $4 \%$ of the annual reporting. This rise in the proportion of reported injuries in Indiana parallels the rapid growth of participants in this game. Since its inception as a war game in 1981, the sport has burgeoned to over one million players each year in North America. ${ }^{5}$

Given the great growth in popularity of paintball, there appears to be a commensurate increase in eye injuries related to the sport. The most important factor in preventing such injuries is the use of goggles or facemasks with polycarbonate lenses (fig 1), which must be worn at all times during play. Paintball should be played in a controlled environment with referees to guard against participants removing their eyewear during combat. None of the patients in our series wore eye or facial protection at the time of the injury. In prior studies only nine of 63 cases $(14 \%)$, where the presence or absence of eye protection was documented, were wearing protection at the time of injury. ${ }^{25}$ The lack of wear of this protection during unsupervised games or the removal of protection during a game for cleaning of lenses appears to be underlying the vast majority of these injuries.

Supported in part by an unrestricted grant from Research to Prevent Blindness Inc.

1 Sparks JJ. All about paintball. Action Pursuit Games Decem-

2 Zwaan J, Bybee L, Casey P. Eye injuries during training Zwaan J, Bybee L, Casey P. Eye injuries during t
exercises with paint balls. Mil Med 1989;161:720-2.

3 Easterbrook M, Pashby TJ. Eye injuries associated with war games. Can Med Assoc f 1985;133:415-19.

4 Tardif D, Little J, Mercier M, et al. Ocular trauma in war games. Physician and Sportsmedicine 1986;14:91-4.

5 Thach AB, Ward TP, Hollifield RD, et al. Ocular injuries from paintball pellets. Ophthalmology 1999;106:533-7.

6 Morris RE, Witherspoon CD, Kuhn F, et al. Eye Injury Registry of Alabama (preliminary report): demographics and prognosis of severe eye injury. South Med $\mathcal{F}$ 1987;80: 810-16.

7 American Society of Testing and Materials (ASTM). Standard specifications for eye protective devices for paintball sports. F1776-97. West Conshocken, PA: ASTM, August 1997 (19428-2959). 